\title{
El asesoramiento individual y la terapia conductual grupal pueden ayudar a los fumadores a dejar de fumar
}

Individual counseling and group behaviour therapy can help smokers to quit smoking

1) Tratamiento indvidual para dejar de fumar

Tratamiento individualLancaster T, y col. Cochrane Database of Systematic Reviews 2017, Issue 3. Art. No.: CD001292

\section{Fuente de datos}

Se realizó una búsqueda en el Registro Especializado del Grupo Cochrane de Adicción al Tabaco (Cochrane Tobacco Addiction Group).

\section{Selección de estudios}

Se incluyeron los ensayos aleatorizados o cuasi-aleatorizados de pacientes adultos con al menos un brazo de tratamiento consistente en asesoramiento individual cara a cara de un trabajador de la salud, quien no participa en la atención clínica de rutina. El resultado fue la cesación tabáquica en el seguimiento al menos seis meses después del inicio del asesoramiento. Se utilizó la definición más rigurosa de abstinencia en cada ensayo, con o sin validación bioquímica de acuerdo a la disponibilidad de los datos.

\section{Extracción de datos}

Dos autores independientes extrajeron los datos por duplicado.
Se registraron las características de la intervención y la población objetivo, el método de asignación al azar y la exhaustividad del seguimiento. En el análisis, se asumió que los participantes perdidos en el seguimiento continuaron fumando. Se expresaron los efectos como una razón de riesgo (RR) para la cesación. Se evaluó la calidad de la evidencia dentro de cada estudio utilizando la herramienta Cochrane de riesgo de sesgo y el enfoque GRADE.

\section{Resultados principales}

Se incluyeron 49 ensayos con alrededor de 19.000 participantes. De ellos, 33 ensayos compararon el asesoramiento individual con una intervención conductual mínima (ver tabla 1), y 11 estudios compararon el asesoramiento individual intensivo con el consejo breve (ver tabla 2). Ninguno de 5 ensayos que compararon diferentes modelos de consejería de intensidad similar detectó diferencias significativas entre ellas.

Tabla 1. Resumen de resultados principales: asesoramiento individual de un consejero para dejar de fumar (incluyendo al menos una sesión cara a cara de 10 minutos o más) comparado con contacto mínimo (asesoramiento breve, atención habitual o suministro de materiales de autoayuda).

\begin{tabular}{|c|c|c|c|c|c|}
\hline \multirow[b]{2}{*}{ Resultados } & \multicolumn{2}{|c|}{$\begin{array}{l}\text { Efectos absolutos previstos * } \\
\qquad(95 \% \text { IC) }\end{array}$} & \multirow[b]{2}{*}{$\begin{array}{l}\text { Riesgo Relativo } \\
\text { (IC 95\%) }\end{array}$} & \multirow[b]{2}{*}{$\begin{array}{l}\mathrm{N}^{0} \text { de } \\
\text { participantes } \\
\text { (Estudios) }\end{array}$} & \multirow[b]{2}{*}{$\begin{array}{l}\text { Calidad de la } \\
\text { evidencia } \\
\text { (GRADE) }\end{array}$} \\
\hline & $\begin{array}{l}\text { Personas que } \\
\text { dejan de fumar } \\
\text { en el grupo } \\
\text { control }\end{array}$ & $\begin{array}{l}\text { Personas que } \\
\text { dejan de fumar } \\
\text { después del } \\
\text { asesoramiento } \\
\text { individual } \\
(95 \% \text { IC) }\end{array}$ & & & \\
\hline \multirow{2}{*}{$\begin{array}{l}\text { Cese del hábito de fumar en } \\
\text { el segui-miento más } \\
\text { prolongado - } 6 \text { meses o más } \\
\text { Sin farmacoterapia sistemática }\end{array}$} & \multicolumn{2}{|c|}{ Población del estudio } & \multirow[b]{2}{*}{$1,57(1,40$ a 1,77$)$} & \multirow[b]{2}{*}{$\begin{array}{c}11.100 \\
(27 \mathrm{ECA})\end{array}$} & \multirow[b]{2}{*}{ 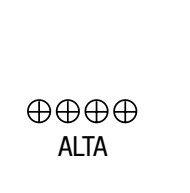 } \\
\hline & 7 por 100 & $\begin{array}{c}11 \text { por } 100 \\
(10 \text { a } 12)\end{array}$ & & & \\
\hline $\begin{array}{l}\text { Farmacoterapia ofrecida a } \\
\text { todos los participantes }\end{array}$ & 11 por 100 & $\begin{array}{c}13 \text { por } 100(11 \\
\text { a } 16)\end{array}$ & $1,24(1,01$ a 1,51$)$ & $\begin{array}{c}2662 \\
(6 \mathrm{ECA})\end{array}$ & $\begin{array}{l}\oplus \oplus \oplus \ominus \\
\text { MODERADA }\end{array}$ \\
\hline \multicolumn{6}{|c|}{$\begin{array}{l}\text { *El riesgo en el grupo de intervención se basa en el riesgo asumido en el grupo de comparación y el efecto relativo de la intervención (y su IC 95\%). } \\
\text { IC: Intervalo de confianza. ECA: Ensayo clínico aleatorizado. } \\
\text { GRADE } \\
\text { - Alta calidad: Estamos muy seguros de que el verdadero efecto se encuentra cerca de la estimación del efecto. } \\
\text { - Calidad moderada: Tenemos una confianza moderada en la estimación del efecto: es probable que el efecto real esté próximo a la estimación del } \\
\text { efecto, pero existe la posibilidad de que sea sustancialmente diferente. }\end{array}$} \\
\hline
\end{tabular}


Tabla 2. Resumen de resultados: Consejería individual intensiva comparado con consejo individual breve.

\begin{tabular}{|c|c|c|c|c|c|}
\hline \multirow[b]{2}{*}{ Resultados } & \multicolumn{2}{|c|}{$\begin{array}{l}\text { Efectos absolutos previstos * } \\
\qquad(95 \% \mathrm{IC})\end{array}$} & \multirow[b]{2}{*}{$\begin{array}{l}\text { Riesgo Relativo } \\
\quad \text { (IC 95\%) }\end{array}$} & \multirow[b]{2}{*}{$\begin{array}{c}\mathrm{N}^{0} \mathrm{de} \\
\text { participantes } \\
\text { (Estudios) }\end{array}$} & \multirow[b]{2}{*}{$\begin{array}{l}\text { Calidad de la } \\
\text { evidencia } \\
\text { (GRADE) }\end{array}$} \\
\hline & $\begin{array}{l}\text { Personas que } \\
\text { dejan de fumar } \\
\text { en el grupo } \\
\text { control }\end{array}$ & $\begin{array}{l}\text { Personas que } \\
\text { dejan de fumar } \\
\text { después del } \\
\text { asesoramiento } \\
\text { individual }\end{array}$ & & & \\
\hline \multirow{4}{*}{$\begin{array}{l}\text { Abandono del } \\
\text { tabaco al } \\
\text { seguimiento más } \\
\text { prolongado }\end{array}$} & \multicolumn{2}{|c|}{ Sin farmacoterapia } & \multirow{4}{*}{$1,29(1,09$ a 1,53$)$} & \multirow{4}{*}{$\begin{array}{c}2920 \\
(11 \mathrm{ECA})\end{array}$} & \multirow{4}{*}{ 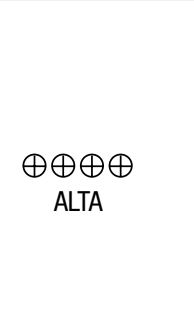 } \\
\hline & 9 por $100^{1}$ & $\begin{array}{l}12 \text { por } 100 \\
(10 \text { a } 14)\end{array}$ & & & \\
\hline & \multicolumn{2}{|c|}{ Con la farmacoterapia } & & & \\
\hline & 14 por $100^{2}$ & $\begin{array}{l}18 \text { por } 100 \\
(15 \text { a } 21)\end{array}$ & & & \\
\hline \multicolumn{6}{|c|}{$\begin{array}{l}\text { * El riesgo en el grupo de intervención (y su IC 95\%) se basa } \\
\text { (y su IC 95\%). }\end{array}$} \\
\hline \multicolumn{6}{|c|}{ IC: Intervalo de confianza. ECA: Ensayo clínico aleatorizado. } \\
\hline \multicolumn{6}{|l|}{ GRADE } \\
\hline \multicolumn{6}{|c|}{ - Alta calidad: Estamos muy seguros de que el verdadero efecto se encuentra cerca de la estimación del efecto. } \\
\hline \multicolumn{6}{|c|}{$\begin{array}{l}\text { - Calidad moderada: Tenemos una confianza moderada en la estimación del efecto: es probable que el efecto real esté próximo a la estimación del } \\
\text { efecto, pero existe la posibilidad de que sea sustancialmente diferente. }\end{array}$} \\
\hline \multicolumn{6}{|c|}{$\begin{array}{l}\text { - Baja calidad: Nuestra confianza en la estimación del efecto es limitada: El verdadero efecto puede ser sustancialmente diferente de la estimación } \\
\text { del efecto. }\end{array}$} \\
\hline \multicolumn{6}{|c|}{$\begin{array}{l}\text { - Muy baja calidad: Tenemos muy poca confianza en la estimación del efecto: Es probable que el efecto real sea sustancialmente diferente de la } \\
\text { estimación del efecto. }\end{array}$} \\
\hline
\end{tabular}

\section{Conclusiones de los autores}

Existe evidencia de alta calidad de que el asesoramiento personalizado para la cesación tabáquica puede ayudar a los fumadores a dejar de fumar. Hay evidencia de calidad moderada de un beneficio adicional cuando se usa el asesoramiento asociado a la farmacoterapia, y de un asesoramiento más intensivo en comparación con una breve intervención de consejería.

Las intervenciones de consejería que se dan fuera de la atención clínica de rutina, por los consejeros para dejar de fumar, incluyendo educadores de salud y psicólogos, ayudan a las personas tabaquistas a dejar de fumar. Esta revisión sistemática Cochrane encontró evidencia de alta calidad de que el asesoramiento individual aumenta la probabilidad de cesación en comparación con un apoyo menos intensivo. Se estimó que el asesoramiento individual, independientemente de la farmacoterapia, aumentaba el abandono del $40 \%$ al $80 \%$ después de al menos seis meses de seguimiento, basándose en la puesta en común de 27 ensayos con más de 11.000 participantes. Suponiendo que un grupo de control abandone a una tasa del $7 \%$ de una intervención breve, se espera que la provisión de consejería resulte en un $10 \%$ a $12 \%$ de abandono, un aumento absoluto de $3 \%$ a
$5 \%$. Esta estimación se basó en el uso de asesoramiento sin ninguna farmacoterapia. Los seis ensayos que ofrecieron farmacoterapia (típicamente terapia de reemplazo de nicotina) a todos los participantes tuvieron un efecto menor y menos seguro. Suponiendo un control de la tasa de abandono del $11 \%$ que refleja el beneficio de la medicación, la adición de asesoramiento podría dar lugar a un aumento absoluto de $0 \%$ a $5 \%$. Es posible que el beneficio adicional relativo sea menor cuando las tasas de abandono en el grupo de control ya están aumentadas mediante el uso de una farmacoterapia efectiva, pero el beneficio absoluto del asesoramiento podría ser similar, con o sin farmacoterapia. El asesoramiento individual es un tratamiento establecido para dejar de fumar. La identificación de la intensidad más efectiva y rentable y la duración del tratamiento para diferentes poblaciones de fumadores sigue siendo un área de investigación. Sin embargo, es probable que las diferencias en el efecto relativo sean pequeñas, especialmente cuando se utiliza el asesoramiento junto con la farmacoterapia. Es poco probable que los ensayos pequeños proporcionen pruebas claras de la eficacia a largo plazo. 
2) Tratamientos basados en terapia grupal para dejar de fumar

Stead LF, y col. Cochrane Database of Systematic Reviews 2017, Issue 3. Art. No.: CD001007

\section{Objetivo}

Determinar el efecto de las intervenciones conductuales grupales para lograr la cesación tabáquica largo plazo.

Fuentes de datos

Se realizó una búsqueda en el registro especializado del Grupo Cochrane de Adicción al Tabaco (Cochrane Tobacco Addiction Group), utilizando los términos terapia conductual, terapia cognitiva, psicoterapia en mayo de 2016.

\section{Selección de estudios}

Se incluyeron ensayos aleatorizados que compararon la terapia grupal con las herramientas de autoayuda, el asesoramiento individual, otra o ninguna intervención (incluida la atención habitual o un control de la lista de espera). Se incluyeron ensayos con un mínimo de dos reuniones grupales y un seguimiento al menos seis meses después del inicio del programa. Se excluyeron los ensayos de farmacoterapias en los que se administró la terapia grupal como coadyuvante de las ramas de intervención y control, a menos que tuvieran un diseño factorial.

\section{Extracción de datos}

Dos revisores extrajeron los datos por duplicado de los participantes, las intervenciones proporcionadas al grupo intervención y al grupo control, incluida la duración del programa, la intensidad y los componentes principales, los resultados, el método de aleatorización y el seguimiento. El resultado principal fue la abstinencia después de al menos seis meses de iniciado el tratamiento. Se utilizó la definición más rigurosa de abstinencia en cada ensayo, con o sin validación bioquímica de acuerdo a la disponibilidad de los datos. Se evaluó la calidad de la evidencia dentro de cada estudio utilizando la herramienta Cochrane de riesgo de sesgo y el enfoque GRADE.

\section{Resultados principales}

Los resultados principales se resumen en la tabla 1.

Tabla 1. Resumen de los hallazgos para el resultado principal de la revisión: abstinencia a los 6 meses, para cada comparación evaluada.

\begin{tabular}{|c|c|c|c|c|c|}
\hline \multirow{3}{*}{ Comparaciones } & \multicolumn{2}{|c|}{$\begin{array}{l}\text { Efectos absolutos* } \\
\qquad(95 \% \text { IC) }\end{array}$} & \multirow{3}{*}{$\begin{array}{l}\text { Riesgo relativo } \\
\text { (IC 95\%) }\end{array}$} & \multirow{3}{*}{$\begin{array}{c}\mathrm{N}^{0} \text { de } \\
\text { participantes } \\
\text { (Estudios) }\end{array}$} & \multirow{3}{*}{$\begin{array}{l}\text { Calidad de la } \\
\text { evidencia } \\
\text { (GRADE) }\end{array}$} \\
\hline & \multirow{2}{*}{$\begin{array}{c}\text { Grupo } \\
\text { control } \\
\begin{array}{c}\text { Personas que } \\
\text { dejan de fumar }\end{array}\end{array}$} & \multirow{2}{*}{$\begin{array}{c}\text { Programa } \\
\text { grupal }\end{array}$} & & & \\
\hline & & & & & \\
\hline $\begin{array}{l}\text { Grupal vs. } \\
\text { autoayuda }\end{array}$ & 5 por 100 & $\begin{array}{l}9 \text { por } 100 \\
(8 \text { a 12) }\end{array}$ & $\begin{array}{c}1,88 \\
(1,52 \mathrm{a} 2,33)\end{array}$ & $\begin{array}{c}4.395 \\
(13 \mathrm{ECA})\end{array}$ & $\begin{array}{l}\oplus \oplus \oplus \ominus \\
\text { MODERADA }\end{array}$ \\
\hline $\begin{array}{l}\text { Grupal vs. consejo } \\
\text { breve }\end{array}$ & 5 por 100 & $\begin{array}{l}6 \text { por } 100 \\
(5 \text { a } 7)\end{array}$ & $\begin{array}{c}1,25 \\
(1,07 \text { a } 1,46)\end{array}$ & $\begin{array}{c}7.601 \\
(16 \mathrm{ECA})\end{array}$ & $\bigoplus \underset{\text { BAJA }}{\oplus \ominus \ominus}$ \\
\hline $\begin{array}{l}\text { Grupal vs. } \\
\text { intervención individual }\end{array}$ & 11 por 100 & $\begin{array}{c}11 \text { por } 100 \\
(8 \text { a } 14)\end{array}$ & $\begin{array}{c}0,99 \\
(0,76 \text { a } 1,28)\end{array}$ & $\begin{array}{c}980 \\
(6 \mathrm{ECA})\end{array}$ & $\begin{array}{l}\oplus \oplus \oplus \ominus \\
\text { MODERADA }\end{array}$ \\
\hline $\begin{array}{l}\text { Grupal + fármacos vs. } \\
\text { apoyo breve }\end{array}$ & 18 por 100 & $\begin{array}{c}20 \text { por } 100 \\
(16 \text { a } 24)\end{array}$ & $\begin{array}{c}1,11 \\
(0,93 \text { a } 1,33)\end{array}$ & $\begin{array}{c}1.523 \\
(5 \mathrm{ECA})\end{array}$ & $\begin{array}{l}\oplus \oplus \oplus \ominus \\
\text { MODERADA }\end{array}$ \\
\hline $\begin{array}{l}\text { Grupal vs. no } \\
\text { intervención }\end{array}$ & 5 por 100 & $\begin{array}{l}13 \text { por } 100 \\
(9 \text { a } 19)\end{array}$ & $\begin{array}{c}2,6 \\
(1,8 \text { a } 3,76)\end{array}$ & $\begin{array}{c}1.098 \\
(9 \mathrm{ECA})\end{array}$ & $\underset{\text { BAJA }}{\oplus \ominus \ominus}$ \\
\hline $\begin{array}{l}\text { * El riesgo en el grupo de } \\
\text { IC: Intervalo de confianza. } \\
\text { GRADE } \\
\text { - Alta calidad: Estamos r } \\
\text { - Calidad moderada: Ten } \\
\text { efecto, pero existe la posi } \\
\text { - Baja calidad: Nuestra c c } \\
\text { del efecto. } \\
\text { - Muy baja calidad: Tene } \\
\text { estimación del efecto. }\end{array}$ & $\begin{array}{l}\text { seguros de que el } \\
\text { ad una confianza } n \\
\text { anza en la estima } \\
\text { muy poca confiar }\end{array}$ & $\begin{array}{l}\text { verdadero efecto } \\
\text { toderada en la es } \\
\text { tancialmente difer } \\
\text { ción del efecto es } \\
\text { iza en la estimaci }\end{array}$ & $\begin{array}{l}\text { ncuentra cerca de } \\
\text { ión del efecto: es } \\
\text { ada: El verdadero } \\
\text { el efecto: Es proba }\end{array}$ & $\begin{array}{l}\text { ción del efecto. } \\
\text { que el efecto rea } \\
\text { de ser sustancia } \\
\text { efecto real sea }\end{array}$ & $\begin{array}{l}\text { רción (y su IC 95\%). } \\
\text { a la estimación del } \\
\text { te de la estimación } \\
\text { te diferente de la }\end{array}$ \\
\hline
\end{tabular}




\section{Conclusiones de los autores}

La terapia grupal es más efectiva que la autoayuda y otras intervenciones menos intensivas. No se encontró evidencia suficiente para evaluar si la terapia grupal es más efectiva o rentable que el tratamiento individual intensivo. No se encontró tampoco evidencia suficiente para respaldar el uso de componentes psicológicos particulares en los programas, más allá del apoyo y el entrenamiento en habilidades.

\section{Comentario}

El Grupo Antitabaco del Hospital Italiano (GRANTAHI) trabaja con pacientes tabaquistas desde hace más de 17 años. La experiencia de nuestro equipo siempre nos ha orientado a priorizar el acompañamiento empático con las personas que se nos acercan a solicitar ayuda. Por otro lado, y en concordancia con estudios previos, ponemos énfasis no solo en trabajar con la motivación del paciente sino también en lograr un periodo de intervención variable entre los 90 y los 300 minutos, en 4 a 9 consultas.

Los tratamientos intensivos para la cesación tabáquica, tanto en su modalidad individual o como grupal, son herramientas efectivas. Parecería ser que a mayor contacto del equipo terapéutico con las personas fumadoras, mayor es la proporción observada de quienes logran mantener la abstinencia. A partir de la evidencia resumida en estas revisiones sistemáticas, no se ha podido demostrar que una modalidad sea mejor que la otra. Una de las fortalezas de los tratamientos grupales es que brindan la oportunidad a las personas fumadoras de interactuar con pares y generar un compromiso con el grupo.

Por otro lado, el tratamiento farmacológico parece disminuir la diferencia entre ambas intensidades de intervención individual, ya que el beneficio observado fue más pequeño en intervenciones intensivas cuando en ambas se usa fármacos.

En la experiencia de nuestro equipo, aun con el uso de fármacos, las intervenciones intensivas tienen un rédito mayor, aunque podría ser un sesgo propio de la especialidad. Cabe aclarar que los fármacos estudiados principalmente en estas revisiones sistemáticas se basan en la terapia de reemplazo nicotínico.

\section{Conclusiones de los comentadores}

Prestar espacio y tiempo a los pacientes tabaquistas para dejar de fumar aumenta considerablemente la posibilidad de abandono y permanencia de la cesación a los 6 meses. La adicción de fármacos (en especial, la terapia de reemplazo nicotínico) aumenta esa posibilidad, en tanto y en cuanto la consejería individual brindada a los pacientes sea intensiva. Por otro lado, los tratamientos intensivos grupales, con 6 a 8 reuniones, también son una herramienta efectiva para trabajar la adquisición de habilidades para manejar la abstinencia, resolver problemas, prevenir las recaídas y modificar conductas.

Mariano Bertoncini [Servicio de Medicina Familiar y Comunitaria y GRANTAHI, Hospital Italiano de Buenos Aires mariano.bertoncini@hospitalitaliano. org.ar] y Silvia Martin [Servicio de Clínica Médica y GRANTAHI, Hospital Italiano de Buenos Aires silvia.martin@ hospitalitaliano.org.ar]

Bertoncini M y Martin S. El asesoramiento individual y la terapia conductual grupal pueden ayudar a los fumadores a dejar de fumar. Evid Act Pract Ambul. 2018;21(1):36-39. Resumido y comentado de: Lancaster T, y col. Individual Behavioural counselling for smokingcessation Cochrane Database of Systematic Reviews 2017, Issue 3. Art. No.: CD001292, y Stead LF, y col. Group behaviour therapy programmes for smoking cessation. Cochrane Database of Systematic Reviews 2017, Issue 3. Art. No.: CD001007.

\section{Referencias Bibliográficas}

1. Fiore MC, y col. Treating Tobacco Use and Dependence. Clinical Practice Guideline. Rockville, MD: U.S. Department of Health and Human Services, Public Health Service. June 2000 .

2. Fiore MC, y col. Treating Tobacco Use and Dependence. Clinical Practice Guideline. An update. US Department of Health and Human Services, Public Health Service. 2008 3. Abordaje integral del tabaquismo /Gabriel González $1^{\circ}$ ed. CABA: Ministerio de Salud de la Nación. REDES 2016 